\title{
Autophagy inhibition facilitates erlotinib cytotoxicity in lung cancer cells through modulation of endoplasmic reticulum stress
}

\author{
ZHONGLIANG WANG ${ }^{1}$, TINGTING DU ${ }^{2}$, XIAORONG DONG ${ }^{1}$, \\ ZHENYU LI $^{1}$, GANG WU ${ }^{1}$ and RUIGUANG ZHANG ${ }^{1}$ \\ ${ }^{1}$ Cancer Center, Union Hospital, Tongji Medical College, Huazhong University of Science and Technology; \\ ${ }^{2}$ Department of Endocrinology, Wuhan Medical and Health Center for Women and Children, Wuhan, Hubei, P.R. China
}

Received January 27, 2016; Accepted March 4, 2016

DOI: $10.3892 /$ ijo.2016.3468

\begin{abstract}
Epidermal growth factor receptor tyrosine kinase inhibitors (EGFR-TKIs) have revolutionized the treatment for non-small cell lung cancer patients, but acquired resistance limit the efficiency of this treatment. As a homeostatic cellular recycling mechanism, autophagy has been proposed to participate in the EGFR-TKI resistance. However, the role of autophagy in lung cancer treatment and the underlying mechanisms have not been clarified. In this study, we found the sensitivity to erlotinib, a well-used EGFR-TKI, was correlated with basal autophagy level. Erlotinib was able to induce autophagy not only in TKI-sensitive, but also TKI-resistant cancer cells. Inhibition of autophagy significantly enhanced cytotoxicity of erlotinib in TKI-resistant cancer cells via modulation of endoplasmic reticulum (ER) stress induced apoptosis. In this process, CCAAT/enhancer binding protein homologous protein (CHOP) acted as an executioner. Downregulation of CHOP with siRNA blocked the autophagy inhibition and erlotinib co-treatment induced apoptosis and prevented cancer cells from this co-treatment-induced cell death. Our findings suggest that autophagy inhibition overcomes erlotinib resistance through modulation of ER stress mediated apoptosis.
\end{abstract}

\section{Introduction}

Non-small-cell lung cancer (NSCLC) is still the leading cause for cancer related mortality worldwide $(1,2)$. Treatment of EGFR-mutant NSCLC patients with specific tyrosine kinase inhibitors (TKIs), such as gefitinib, erlotinib or afatinib, has led to remarkable tumor shrinkage and improvement in progression-free survival and quality of life compared with the

Correspondence to: Dr Gang Wu or Dr Ruiguang Zhang, Cancer Center, Union Hospital, Tongji Medical College, Huazhong University of Science and Technology, Wuhan, Hubei, P.R. China

E-mail: xhzlwg@163.com

E-mail: zrg27@163.com

Key words: lung cancer, erlotinib, autophagy, chop, endoplasmic reticulum stress standard chemotherapy (3-5). However, not all patients respond to TKI treatment, and patients who initially benefited from the TKI treatment inevitably developed drug resistance $(6,7)$. The resistance mechanisms to TKI treatment can be grouped into four categories: mutation of EGFR to a drug-resistance state, for example, T790M mutation; activation of a bypass pathway, such as MET amplification; impairment of a pathway that is essential for TKI-induced apoptosis; and histologic transformation to small cell lung cancer or epithelial-mesenchymal transition (8). Apart from these clues, accumulating evidence indicate that macroautophagy (hereafter autophagy) plays a vital role in the resistance to TKI.

Autophagy is a lysosomal degradative pathway characterized by the formation of double-membrane autophagic vesicles, which engulf portions of the cytosol, damaged organelles, protein aggregates and bacteria. In virtually all cells, autophagy occurs at basal levels to maintain homeostatic balance by updating proteins and organelles (9). Autophagy is upregulated when cells are under unfavorable environments, such as starvation, hypoxia, and drug exposure, to help cells survive in adverse conditions.

Cancer cells have increased metabolic demands due to high levels of cellular proliferation and cellular stress, which activates autophagy to maintain energy balance and to prevent cell death (10). Theoretically, autophagy may be a strategy to confer not only unfavorable environments but also chemicals exposure. Many cancer therapeutics, including TKIs, were reported to activate pro-survival autophagy and blockage of autophagy facilitates cell death (11-14). However, so far, the mechanisms underlying the synergistic effect of EGFR-TKI and autophagy inhibition remains unclear.

The endoplasmic reticulum (ER) is an organelle involved in protein folding and assembly, lipid and sterol biosynthesis, and free calcium storage. ER stress is caused when accumulation of unfolded and misfolded proteins exceed the capacity of the ER process. Upon ER stress, protein kinase RNA (PKR)-like ER kinase (PERK) is activated following dissociation from the ER chaperone GRP78. Activated PERK transiently inhibits protein synthesis by phosphorylating eukaryotic initiation factor $2 \alpha$ (eIF $2 \alpha)$. Activation of PERK can also lead to the induction of CCAAT/enhancer binding protein homologous protein (CHOP), which switches the ER stress response from proadaptive to proapoptotic signaling (15-17). Given inhibition 
of autophagy would cause accumulation of damaged proteins and excessive accumulation of misfolded proteins would induce ER stress, we reasoned that there may be a link between autophagy inhibition and ER stress induced apoptosis.

In this study, we tested our hypothesis by a combination of western blotting, transmission electron microscopy, and fluorescent microscopy to detect autophagy in several types of cancer cells after erlotinib treatment. We also explored the mechanisms underlying the synergistic effect of autophagy inhibition and erlotinib. Our findings suggest that autophagy acts as a cell protect factor and contributes to the EGFR-TKI resistance. The combination of autophagy inhibition and erlotinib may be a promising route to enhance EGFR-TKI sensitivity and overcome EGFR-TKI resistance.

\section{Materials and methods}

Reagents and antibodies. The chemicals used were erlotinib (Selleck), chloroquine (CQ, Sigma), and 3-methyladenine (3-MA, Sigma). Erlotinib was dissolved in dimethylsulfoxide (DMSO), while CQ and 3-MA were dissolved in cells culture medium. The primary antibodies were antibodies against LC-3 (1:1,000, Novus, \#NB100-2220), beclin-1 (1:1,000, Cell Signaling Technology, \#3495), caspase 3 (1:500, ProteinTech, \#19677-1-AP), cleaved-caspase 3 (1:500, Cell Signaling Technology, \#9664), chop (1:1,000, Cell Signaling Technology, \#2895), p-EIF2 $\alpha$ (1:1,000, Cell Signaling Technology, \#3398), EIF2 $\alpha$ (1:1,000, Cell Signaling Technology, \#5324), EGFR (1:1,000, Cell Signaling Technology, \#4267) and GAPDH (1:2,000, Cell Signaling Technology, \#5174).

Cell culture. The human NSCLC cell lines HCC827 (adenocarcinoma), A549 (adenocarcinoma), H460 (large cell carcinoma) and H1975 (adenocarcinoma) were obtained from Cell Bank (Chinese Academy of Sciences) and maintained in suggested complete medium. HCC 827 has a mutation in the EGFR tyrosine kinase domain (E746-A750 deletion). H1975 has two mutations in the EGFR tyrosine kinase domain (L858R and T790M). While A549 and H460 are EGFR wild-type cells. To establish an EGFR-TKI-resistant cell line, HCC827 cells were cultured in medium containing escalating concentrations of erlotinib for 6 months to select cells which could grow in micromolar concentrations of erlotinib. The selected cells were confirmed erlotinib-resistant, and named HCC827-R.

Cell proliferation. The cytotoxicity of chemicals against cancer cells were determined with CCK8 tests. Cells were seeded in 96-well plates at a concentration of 1,500-2,000 cells per well, allowed to attach overnight, and treated with various concentrations of chemicals. After 72-h incubation, $10 \mu \mathrm{l}$ of CCK8 (Boster, China) was added into each well for another 1-h incubation. Then, the cell optical density (OD) was measured at an absorbance wavelength of $450 \mathrm{~nm}$.

Clonogenic assay. Cells were seeded in 6-well plates at a concentration of 1,000 cells per well, allowed to attach overnight, and treated with $10 \mu \mathrm{M} \mathrm{CQ}, 0.1 \mu \mathrm{M}$ erlotinib, $1 \mu \mathrm{M}$ erlotinib, alone or in combination. Culture medium and chemicals were replaced every 72 h. After 10-14 days, cells were fixed with methanol and stained with $1 \%$ crystal violet. Colonies were counted with ImageJ software.

Western blotting. After chemical treatment, cells were lysed in a lysis buffer containing protease and phosphatase inhibitors. Equal amounts of protein were separated by SDS-PAGE and transferred to PVDF membrane (Millipore, Billerica, MA, USA). After blocking with $5 \%$ non-fat milk, the membranes were then incubated overnight at $4^{\circ} \mathrm{C}$ with primary antibodies. Then, after washing with TBST, the membranes were incubated with corresponding secondary antibodies conjugated with horseradish peroxidase, developed with ECL solution, and exposed onto Hyperfilm (OptiCam600; UVP, USA).

cDNA and siRNA transfection. The GFP-tagged LC3 cDNA expression construct was kindly provided by Professor Jinghua Ren (Union Hospital, Wuhan, China) and transfected with Lipofectamine 2000 (Invitrogen). The CHOP-siRNA and non-specific control of siRNA were from RiboBio Technology (Guangzhou, China) and the targeted sequence of CHOP-siRNA was GGCTCAAGCAGGAAATCGA. Cells were transfected with siRNA using Interferin (Plosplus) according to the manufacturer's instructions and incubated $24 \mathrm{~h}$ prior to further treatments.

Apoptosis assay. Apoptosis events were measured using FITC Annexin V Apoptosis Detection kit (BD) that quantitatively measures Annexin V or PI (propidium iodide)-positive cells. Western blot analyses were used to detect the protein level of cleaved caspase 3 after various treatments.

Transmission electron microscopy. Transmission electron microscopy was performed as previously described (18). In brief, $\mathrm{H} 1975$ cells were treated with erlotinib $(1 \mu \mathrm{M})$ for $48 \mathrm{~h}$, washed 3 times with PBS, and fixed with glutaraldehyde overnight. After washing with PBS, cells were fixed in $1 \%$ $\mathrm{OsO}_{4}$ and embedded in polybed resin. The ultrathin sections were doubly stained with uranyl acetate and lead citrate and analyzed by transmission electron microscopy (Hitachi, Japan).

Quantification and statistics. Differences between groups were compared using the Student's t-test or two-way ANOVA followed by the Bonferroni test. The criterion for statistical significance was $\mathrm{P}<0.05$.

\section{Results}

Autophagy involves EGFR-TKI sensitivity. With CCK8 tests, we examined the sensitivity of HCC827, A549, H460, H1975, and HCC827-R cells to erlotinib. HCC827 cells, with a mutation in the EGFR tyrosine kinase domain (E746-A750 deletion), was the most sensitive to erlotinb. In contrast, HCC827-R cells showed significant resistance to erlotinib. A549 and H460 cells, both of EGFR wild-type, were relatively resistant to erlotinib. H1975 cells, with two mutations in the EGFR tyrosine kinase domain (L858R and T790M), showed further resistance to erlotinb than A549 or H460 cells (Fig. 1A and B). In western blotting results, HCC827 cells showed abundant basal EGFR expression, while the basal EGFR 

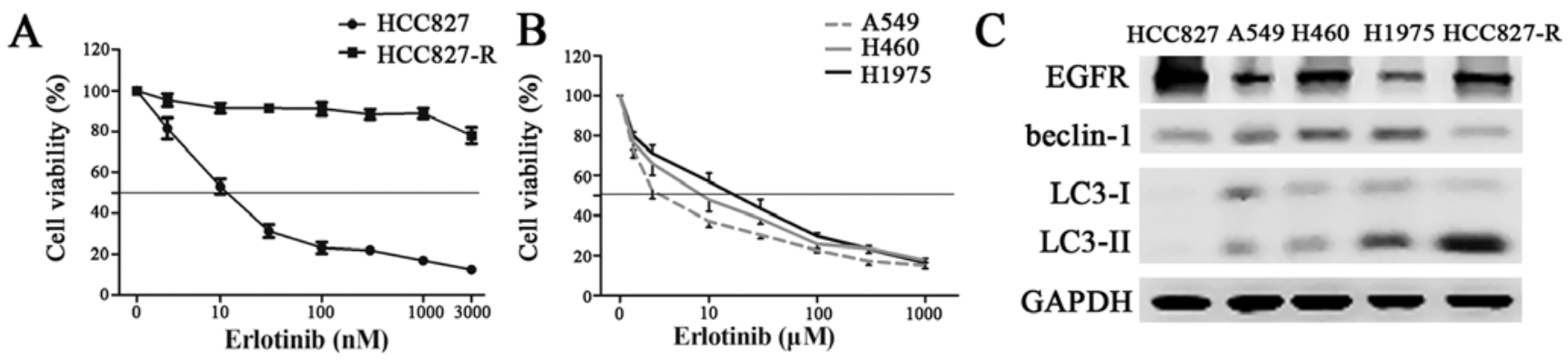

Figure 1. The sensitivity to erlotinib and basal autophagy level of several NSCLC cell lines. (A and B) HCC827 (E746-A750 deletion), HCC827-R (an erlotinibresistant cell line developed from HCC827 by constant exposure of erlotinib for 6 months), A549 (wtEGFR), H460 (wtEGFR) and H1975 (L858R and T790M) cells were treated with increasing concentrations of erlotinib for $72 \mathrm{~h}$ before CCK8 tests. Data are means \pm SEM of three independent experiments. (C) Western blotting showed the basal levels of EGFR, beclin-1, LC3-I and LC3-II of these five cell lines.

A

HCC827

A549

$\mathrm{H} 1975$
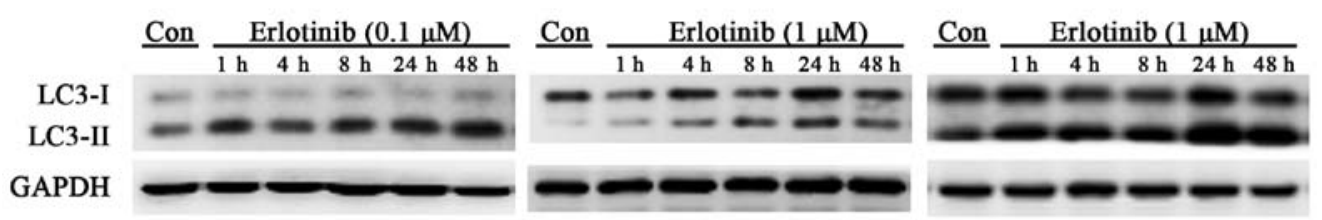

B
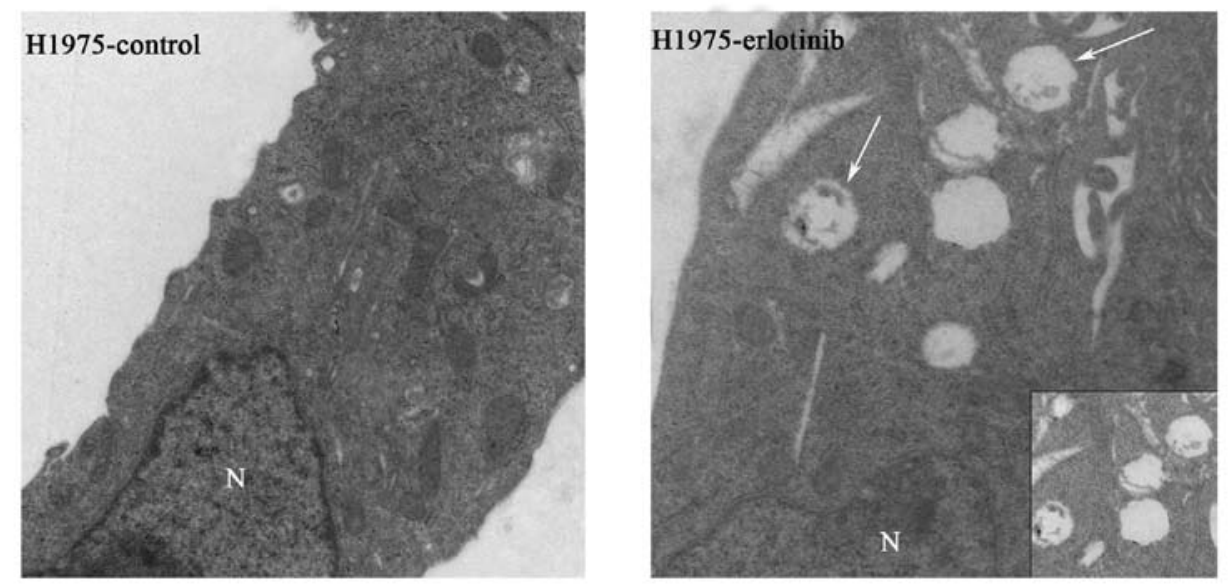

Figure 2. Erlotinib induces autophagy in NSCLC cell lines. (A) HCC827, A549, and H1975 cells were treated with erlotinib (0.1 $\mu$ M for HCC827, $1 \mu$ M for A549 and H1975) for indicated times. Western blotting showed that erlotinib induced a time-dependent increase of LC3-II in all three cell lines. (B) Photographs from transmission electron microscopy showed autophagosomes (indicated by arrows) in H1975 cells after 48-h treatment with $1 \mu \mathrm{M}$ erlotinib. N, nucleus.

expression of A549, H460 and HCC827-R cells was moderate. H1975 cells showed only slight EGFR expression. We tested the basal autophagy level by measuring the conversion of the LC3/Atg8 from the cytoplasmic form LC3-I, to the autophagosomic form LC3-II. The results showed that HCC827-R cells expressed considerable level of LC3-II, indicating an active basal autophagy activity. $\mathrm{H} 1975$ cells also showed easily detectable amounts of conversion from LC3-I to LC3-II, while the other three cells showed little conversion (Fig. 1C). In line with the expression of LC3-II, HCC827, A549, H460, and H1975 cells showed increasing protein level of beclin-1, which was negatively correlated with the cell sensitivity to erlotinib. However, HCC827-R cells showed slight beclin-1 expression, similarly to HCC827 cells. Given that autophagy is an inducible process under survival stress and the active autophagy activity of HCC827-R and H1975 was generated in a wellcultured environment, just like the other three cell lines, we reasoned that the high basal autophagy levels of HCC827-R and H1975 were not stress-induced, but an intrinsic character and involved in their resistance to erlotinib.

Autophagy is induced in cancer cells after erlotinib treatment. Autophagy was induced under treatment of erlotinib in three cell lines, HCC827, A549, and H1975. With HCC827 cells, $0.1 \mu \mathrm{M}$ of erlotinib induced marked autophagy just $1 \mathrm{~h}$ after exposure and lasted for $>48 \mathrm{~h}$. Of note, after $4 \mathrm{~h}$ of erlotinib exposure, HCC827 cells showed less conversion of LC3 compared with that of 1-h exposure, but then the conversion of LC3 turned to increase from 4 to $48 \mathrm{~h}$ persistently. With A549 cells, $1 \mu \mathrm{M}$ of erlotinib induced a time-dependent accumulation of autophagy, with the highest level achieved at $24 \mathrm{~h}$. Similar with A549, H1975 cells also showed a time-dependent increase of autophagy under the treatment of erlotinib $(1 \mu \mathrm{M})$ (Fig. 2A). We also measured the punctate fluorescence after 


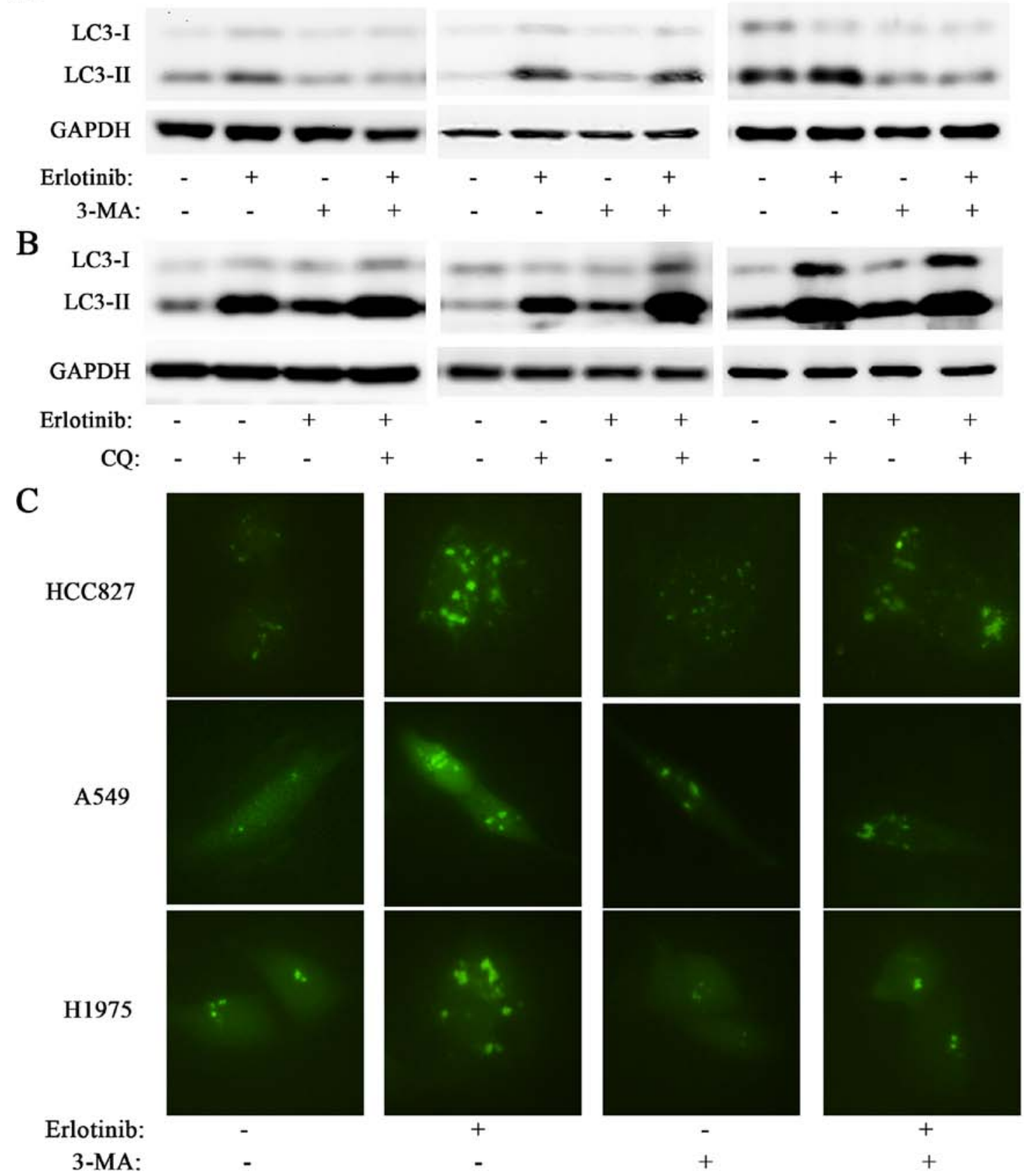

Figure 3. (A) HCC827, A549, and H1975 cells were treated with erlotinib ( $0.1 \mu \mathrm{M}$ for HCC827, $1 \mu \mathrm{M}$ for A549 and H1975), 3-MA (5 mM), or a combination of both for $48 \mathrm{~h}$. Western blotting showed that treatment with 3-MA reversed erlotinib-induced increase of LC3-II in all three cell lines. (B) HCC827, A549, and $\mathrm{H} 1975$ cells were treated erlotinib $(0.1 \mu \mathrm{M}$ for HCC $827,1 \mu \mathrm{M}$ for A549 and $\mathrm{H} 1975)$, chloroquine (CQ, $10 \mu \mathrm{M})$, or a combination of both for $48 \mathrm{~h}$. In all three cell lines, western blotting showed that CQ treatment significantly increased the LC3-II level, while the combination of CQ and erlotinib induced a further accumulation of LC3-II as compared with CQ single treatment. (C) HCC827, A549, and H1975 cells were transfected with a GFP-LC3 construct. In all the three cell lines, photographs from fluorescence microscopy showed that 3-MA reversed the erlotinib-induced punctate fluorescence increase.

erlotinib treatment in the cells transfected with a GFP-tagged LC3 construct. In all three cell lines, erlotinib induced more punctate fluorescence than did the control cells, which showed only slight fluorescence (Fig. 3C). This result was further confirmed with transmission electron microscopy, which revealed autophagosomes in $\mathrm{H} 1975$ cells after 48-h erlotinib treatment, whereas autophagosomes were scarce in untreated cells (Fig. 2B). Together, these findings suggest that erlotinb treatment induces autophagy in both EGFR-TKI-sensitive and -resistant cancer cells.
3-MA and CQ abates erlotinib-induced autophagy. As a classic autophagy inhibitor, 3-MA was tested for the ability to abate erlotinib-induced autophagy. With western blotting, 3-MA was able to diminish the conversion from LC3-I to LC3-II in HCC827, A549, and H1975 cells. The combination of 3-MA and erlotinib markedly decreased the erlotinibinduced conversion from LC3-I to LC3-II, nearly to the same level with 3-MA single treatment (Fig. 3A). This result was further confirmed by measuring the punctate fluorescence in the cells transfected with a GFP-tagged LC3 construct. 

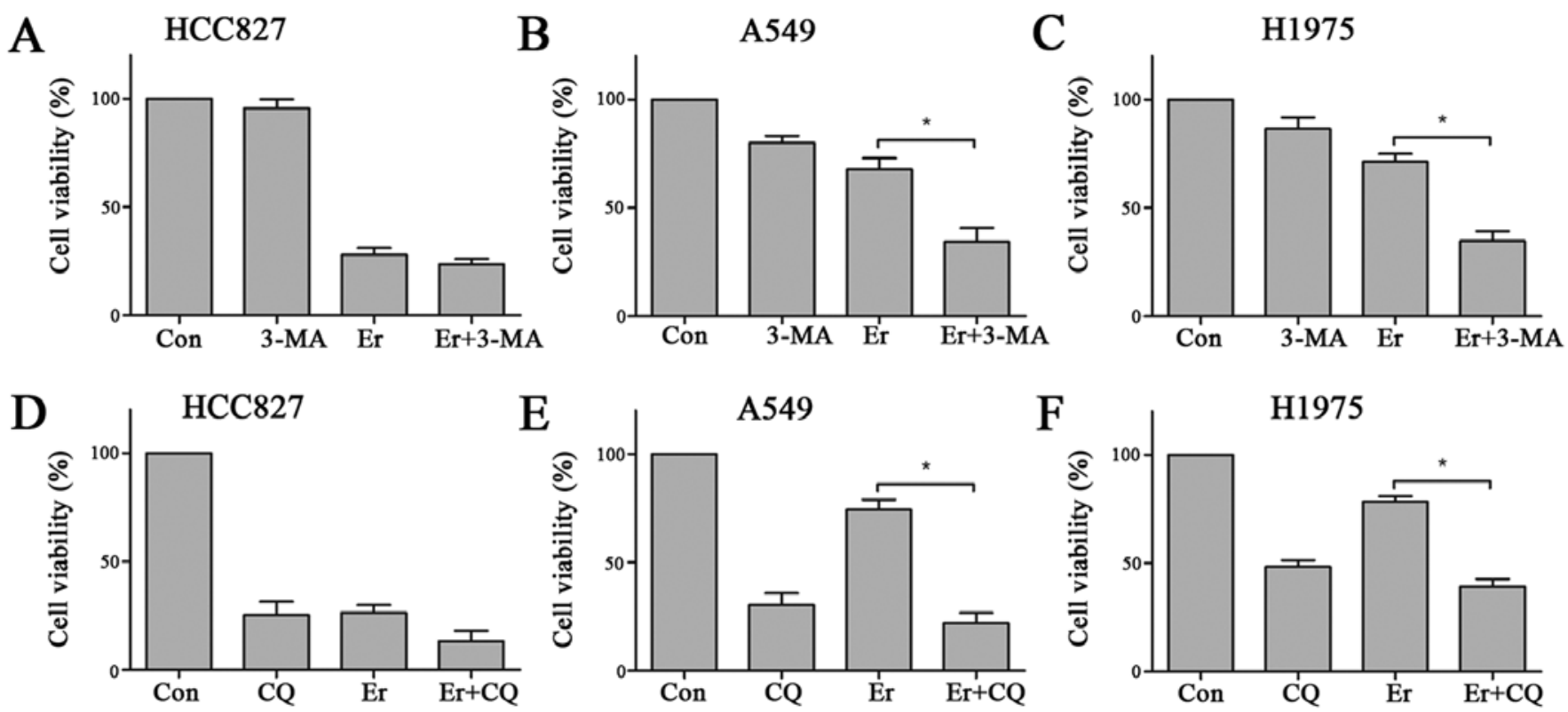

Figure 4. 3-MA and CQ show synergistic cytotoxicity with erlotinib in NSCLC cell lines. (A-C) HCC827, A549, and H1975 cells were exposed to erlotinib $(0.1 \mu \mathrm{M}$ for HCC $827,1 \mu \mathrm{M}$ for A549 and H1975), 3-MA (5 mM), or the combination of both for $72 \mathrm{~h}$ before CCK8 tests. (D-F) The three cell lines were exposed to erlotinib [same concentration with (A-C) above], CQ $(10 \mu \mathrm{M})$, or the combination of both for $72 \mathrm{~h}$ before CCK8 tests. Data are means \pm SEM of three independent experiments. ${ }^{*} \mathrm{P}<0.05$.

In all three cell lines, 3-MA significantly downregulated the erlotinib-induced LC3-GFP protein accumulation (Fig. 3C). We also used CQ, an autophagy-lysosomal inhibitor, to block erlotinib-induced autophagy. While 3-MA is able to inhibit autophagosome formation, CQ functions by blocking the degradation of autophagosomes and thereby induces accumulation of LC3-II. In this experiment, treatment of CQ induced abundant accumulation of LC3-II in all three cell lines. The combination of CQ and erlotinib showed still further LC3-II accumulation than $\mathrm{CQ}$ alone, indicating that $\mathrm{CQ}$ blocked the degradation of erlotinib-induced autophagosomes (Fig. 3B). Moreover, this result illustrates that the erlotinib-induced LC3-II accumulation was a reflection of increased autophay flux, rather than dysfunction of lysosomes.

Combination with autophagy inhibition enhances erlotinibinduced cytotoxicity. As autophagy might be involved in the resistance of $\mathrm{H} 1975$ cells to erlotinib, we tested the synergistic effect of autophagy inhibition and EGFR-TKI by combining 3-MA with erlotinib. With CCK8 tests, 3-MA and erlotinib co-treatment showed significant synergistic cytotoxicity to H1975 cells while 3-MA single treatment showed only slight influence (Fig. 4C). Consistently, A549 cells showed much higher sensitivity to the co-treatment of 3-MA and erlotinib than treatment of 3-MA or erlotinib alone (Fig. 4B). HCC827 was naturally sensitive to erlotinb treatment, but resistant to 3-MA (Fig. 4A). The effect of CQ was examined as well. To our surprise, HCC827, A549, and H1975 cells were all extraordinarily sensitive to CQ. Under CQ $(10 \mu \mathrm{M})$ exposure for $72 \mathrm{~h}$, the cell viabilities decreased to $25.4 \pm 6.2 \%$ for HCC 827 cells, $30.4 \pm 5.3 \%$ for A 549 cells, and $48.5 \pm 2.9 \%$ for $\mathrm{H} 1975$ cells. For H1975 and A549 cells, the combination of CQ and erlotinib showed increased cytotoxicity compared with CQ single exposure, but failed to reach statistical difference. However, when compared with erlotinib single treatment, co-administration of CQ and erlotinib significantly enhanced the inhibition of cell proliferation. For HCC827 cells, erlotinib, with or without $\mathrm{CQ}$, was able to dramatically inhibit the cell proliferation, with the combination treatment showed further significant effect (Fig. 4D-F).

The impact of chemicals on colony formation was further measured. HCC827, A549, and H1975 cells were treated with $10 \mu \mathrm{M} \mathrm{CQ}, 0.1 \mu \mathrm{M}$ erlotinib, $1 \mu \mathrm{M}$ erlotinib, or the combination of CQ and erlotinib for 10-14 days. It was found that $0.1 \mu \mathrm{M}$ erlotinib caused 16 and $15 \%$ reduction of cell colonies in A549 and H1975 cells, respectively. While, $1 \mu \mathrm{M}$ erlotinib, respectively caused 56 and $28 \%$ reduction of cell colonies in A549 and H1975 cells. In HCC827 cells, 0.1 or $1 \mu \mathrm{M}$ erlotinib showed nearly complete elimination of colonies formation. Remarkably, CQ alone or in combination with erlotinib caused nearly complete inhibition of cell growth in all the three cell lines (Fig. 5). These findings indicate that inhibition of autophagy can enhance erlotinib sensitivity in sensitive NSCLC cells and overcome erlotinb resistance in resistant NSCLC cells.

Combination of autophagy inhibition and erlotinib induces ER stress and apoptosis. We tested whether combination of erlotinib and inhibition of autophagy with CQ would induce apoptosis in EGFR-TKI-resistant cells. To this aim, H1975 was treated with CQ, erlotinib, alone or in combination for $48 \mathrm{~h}$, and then measured for apoptosis with flow cytometry by quantitatively measuring Annexin V and PI. The results showed CQ induced statistically significant induction of apoptosis, but erlotinib did not. The combination of CQ and erlotinib also induced apoptosis, and this effect was significantly greater than apoptosis seen with CQ single treatment (Fig. 6A). Consistent with the flow cytometry results, western blot analyses revealed 
A

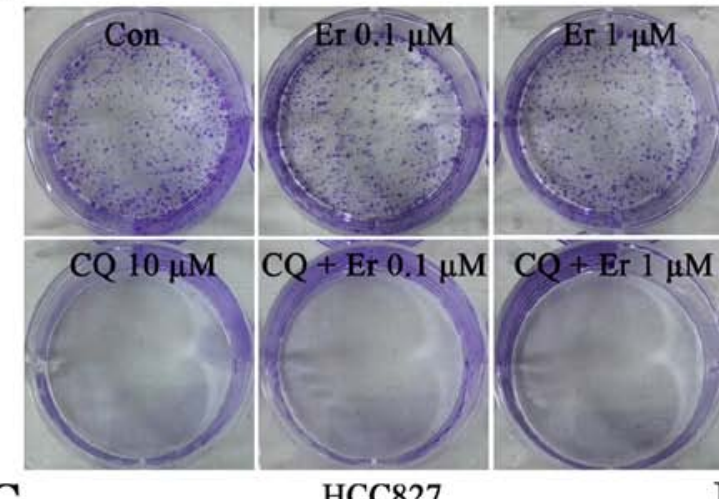

C

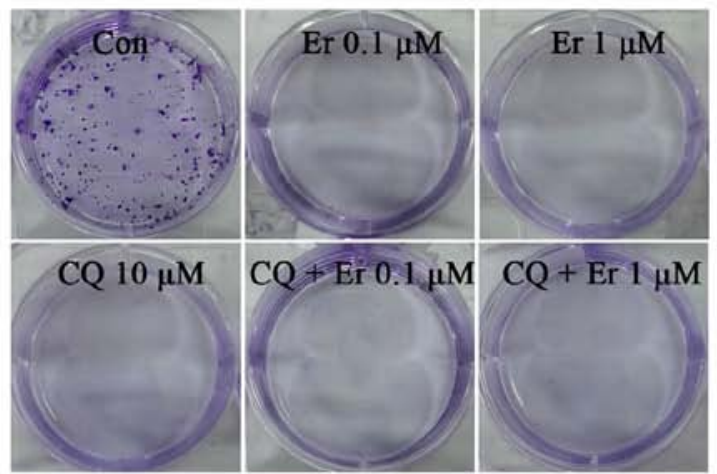

B

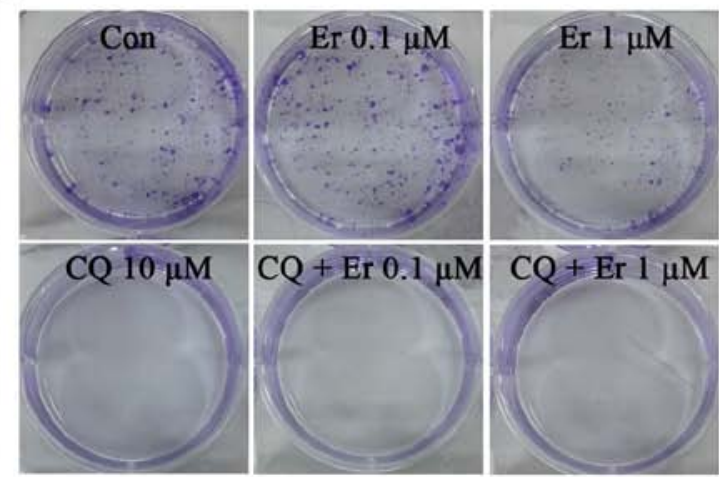

D

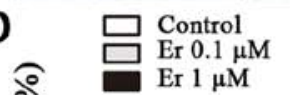

。̊

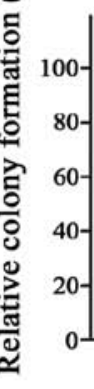

$\mathrm{CQ} 10 \mu \mathrm{M}$

CQ $10 \mu \mathrm{M}+\mathrm{Er} 0.1 \mu \mathrm{M}$

CQ $10 \mu \mathrm{M}+\operatorname{Er} 1 \mu \mathrm{M}$
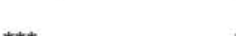

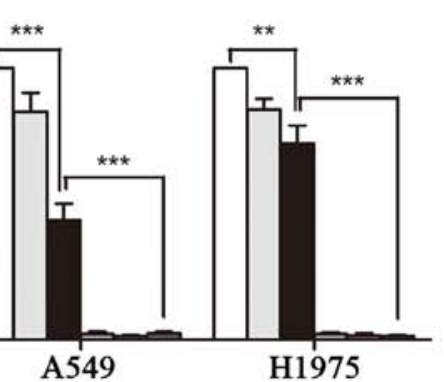

Figure 5. CQ alone or in combination with erlotinib caused nearly complete inhibition of colony formation of NSCLC cell lines. HCC827, A549, and H1975 cells were seeded in 6-well plates and treated with CQ $(10 \mu \mathrm{M})$, erlotinib $(0.1$ and $1 \mu \mathrm{M})$ alone, or in combination for 10-14 days before being stained (A-C) and the number of colonies was quantified (D). Data are means \pm SEM of three independent experiments. ${ }^{* *} \mathrm{P}<0.01,{ }^{* * *} \mathrm{P}<0.001$.

A

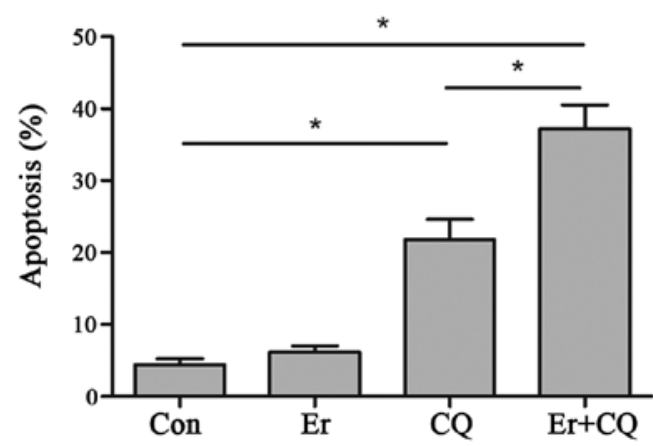

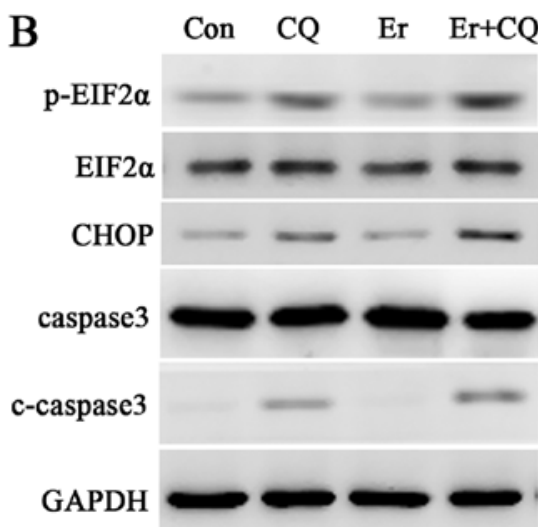

C

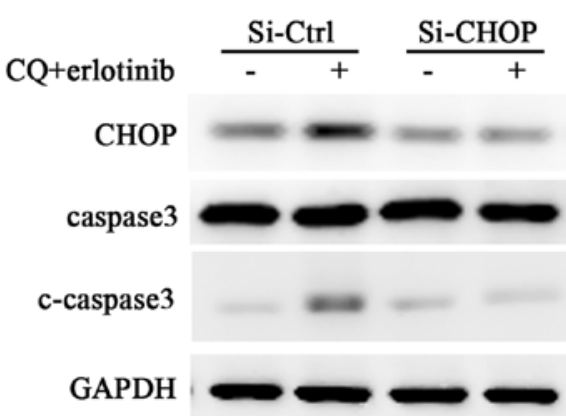

$\mathrm{D}$

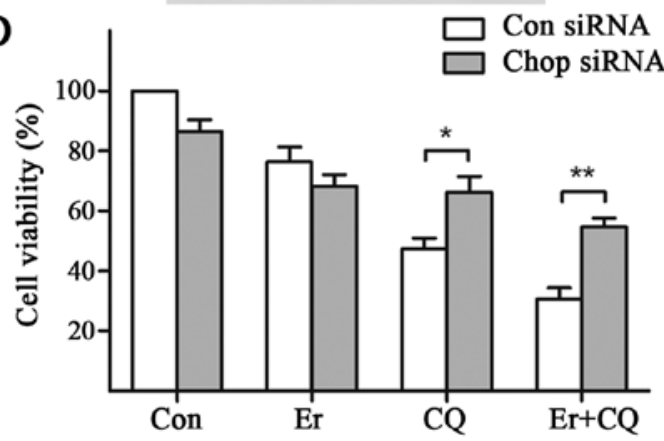

Figure 6. Combination of CQ and erlotinib induced apoptosis through modulation of ER stress. (A) H1975 cells were treated with erlotinib ( $1 \mu \mathrm{M})$, CQ (10 $\mu \mathrm{M})$, or a combination of both for $48 \mathrm{~h}$. Apoptosis was measured with Annexin V and PI double staining followed by flow cytometry analysis. (B) H1975 cells were treated as in (A) above. Western blotting showed that CQ alone, or in combination with erlotinib, induced cleavage of caspase 3 and upregulation of p-eIF2 $\alpha$ and CHOP. (C) H1975 cells were transiently transfected with siRNA against CHOP or control siRNA $24 \mathrm{~h}$ before exposed to co-treatment of CQ and erlotinib or no treatment for $48 \mathrm{~h}$. Western blotting showed that siRNA targeting CHOP abolished CQ and erlotinib co-treatment induced upregulation of CHOP and cleavage of caspase 3, but not in control siRNA treated cells. (D) Compared with control siRNA, siRNA targeting CHOP protected H1975 cells from the cytotoxicity of CQ or CQ combination with erlotinib. Data are means \pm SEM of three independent experiments. ${ }^{*} \mathrm{P}<0.05,{ }^{* * *} \mathrm{P}<0.01$. 
that CQ markedly induced the activation of caspase 3, a critical executioner of apoptosis, which was further enhanced by combination of CQ and erlotinib (Fig. 6B).

The impact of autophagy inhibition and erlotinib on triggering ER stress was analysed by measuring its welldocumented markers, CHOP and the phosphorylated form of the initiation factor $2-\alpha$ (p-eIF $2 \alpha)$. CHOP is an ER-stress associated proapoptotic marker, and eIF $2 \alpha$ is a translation initiation factor phosphorylated by protein kinase RNA-like endoplasmic reticulum kinase (PERK) in response to ER stress. The protein levels of p-eIF $2 \alpha$ and CHOP increased in H1975 cells after treatment with CQ for $48 \mathrm{~h}$, whereas the 48-h treatment of erlotinib showed no change. By contrast, co-administration of $\mathrm{CQ}$ and erlotinib induced a more significant increase of p-eIF2 $\alpha$ and CHOP compared with CQ single treatment (Fig. 6B). These data suggest that $\mathrm{CQ}$ with or without erlotinib treatment could induce apoptosis and trigger ER stress.

CHOP knockdown counteracts the cytotoxicity and apoptosis induced by co-treatment of $C Q$ and erlotinib. To gain further insight into the role of ER stress in CQ and erlotinib co-treatment induced cytotoxicity, the effects of knockdown of CHOP was tested. At $24 \mathrm{~h}$ after CHOP siRNA transfection, H1975 cells was exposed to CQ, erlotinib, or the combination of both chemicals for additional $48 \mathrm{~h}$ for western blot analyses or $72 \mathrm{~h}$ for CCK8 tests. As a result, knockdown of CHOP blocked the CQ-erlotinib co-treatment induced CHOP upregulation. Also, the cleavage of caspase 3 induced by CQ-erlotinib co-treatment was significantly diminished, indicating blockage of apoptosis caused by CQ-erlotinib co-treatment (Fig. 6C). In CCK8 tests, knockdown of CHOP significantly counteracted the cytotoxicity caused by CQ or CQ-erlotinib co-treatment, while the effect of erlotinib single treatment was not obviously affected (Fig. 6D).

\section{Discussion}

In this study, we found that erlotinib induced autophagy in three lung cancer cell lines, HCC827, A549, and H1975 by detecting conversion from LC3-I to LC3-II with western blotting. The GFP-LC3 construct and transmission electron microscopy were used to further confirm the autolysosome formation after erlotinib exposure. Two kinds of autophagy inhibitors, 3-MA and $C Q$, were used to ablate the induction of autophagy under exposure of erlotinib. Synergistic effect between erlotinib and 3-MA/CQ were found in all three cell lines, with CQ single treatment presenting much more cytotoxicity than 3-MA, indicating different mechanisms for blocking autophagy may associate with their distinct cytotoxicity. Co-treatment of CQ and erlotinib induced ER stress in H1975 cells, which evoked apoptosis through modulation of ER stress. Inhibition of CHOP upregulation alleviated apoptosis and counteracted cytotoxicity caused by CQ or CQ-erlotinib co-administration.

EGFR-EKI induces autophagy. Accumulating studies have reported that anticancer therapies, including radiotherapy, chemotherapy and targeted therapies, could induce autophagy in different tumor cell lines (19-22). Though the correlation between anticancer therapies and autophagy has been proposed, the role of autophagy in cancer treatment is complex. On the one hand, the role of autophagy is pro-survival and contributes to therapy resistance through removal of damaged cell components and proteins to help cancer cells survive under stress. On the other hand, autophagy is pro-death and contributes to therapy effect by enhancing induction of apoptosis or mediating 'autophagic cell death'. One theory to explain this double-faced role of autophagy in cancer treatment is that there is a checkpoint in therapy induction of autophagy. As a balance keeper, modest autophagy induction could eliminate damage and recycle energy to promote cancer cells survival. While excessive autophagy may induce vital protein or organelle lysis, triggering apoptosis or autophagic cell death, known as the second programmed cell death.

EGFR-TKIs, was reported to induce marked autophagy in sensitive cancer cells, like HCC827 and PC-9 $(14,18,22)$. In EGFR-TKI-resistant cancer cells, most authors presented that EGFR-TKI was able to induce autophagy, but Li et al reported that EGFR-TKI failed to induce autophagy in resistant cells (23). Apart from targeting EGFR, other TKIs, such as imatinib (targeting BCRABL, KIT, and PDGFR), dasatinib (targeting BCRABL, KIT, and SRC), and sorafenib (a multi-tyrosinekinase inhibitor), could induce autophagy in distinct tumor cell lines as well (24-26). Furthermore, the anti-EGFR antibody cetuximab was reported to induce autophagy through inhibiting the class I PI3K/Akt/mTOR pathway (13). In this study, we found that erlotinib, a well-used EGFR-TKI, elicited a rapid and robust induction of autophagy in HCC827, and caused a relatively slow but persistent induction of autophagy in A549 and H1975 cells, as demonstrated by the increased conversion of LC3. We also found that EGFR-TKI resistance was positively correlated with baseline autophagy level. As compared with the parental HCC827 cells, HCC827-R cells showed significant resistance to erlotinib and much more conversion of LC3. Consistently, A549, H460, and H1975 cells showed increasing conversion of LC3 and exaggerating resistance to erlotinib. In line with this point, Guo et al observed that patients with low LC3 expression had a higher objective response rates in advanced colorectal cancer patients treated with cetuximab-containing chemotherapy, indicating that autophagy may reduce cetuximab efficacy in patients with colorectal cancer (27).

Autophagy and EGFR-TKI resistance. EGFR-TKI acquired resistance is the major failure in treatment of NSCLC, in which half of resistance can be attributed to EGFR T790M mutation (3). However, inhibition of signaling molecules downstream of EGFR did not enhance the sensitivity of NSCLC cells with EGFR T790M mutation to EGFR-TKIs, indicating that there may be another pathway mediating EGFR-TKI resistance $(2,28)$. Several studies demonstrated that autophagy inhibition acted synergistically with EGFR-TKIs to induce lung cancer cells death $(18,22,29)$. For example, Zou et al (18) reported that erlotinib, at a clinically relevant concentration $(2 \mu \mathrm{M})$ induced autophagy in NSCLC cells with wild-type EGFR and inhibition of autophagy with CQ enhanced erlotinib cytotoxicity in vitro and in vivo. In this study, we confirmed the synergistic effect of autophagy inhibition and erlotinib with 3-MA and CQ, two distinct inhibitors of autophagy, in three NSCLC cell lines with different types of EGFR mutation. 
3-MA, a PtdIns3K inhibitor that effectively blocks an early stage of autophagy, reversed erlotinib-induced autophagy, demonstrated by decline of LC3 conversion. 3-MA was able to statistically significantly increase the cytotoxicity of erlotinib in A549 and H1975 cells.

CQ is an FDA approved drug used for malaria, rheumatoid arthritis and other autoimmune diseases, with an established history of generally well-tolerated clinical use. CQ is a weak base with hydrophobic characteristics that diffuses into the lysosomes of cells, where it becomes protonated and trapped, thus leading to increase in lysosomal $\mathrm{pH}$. These CQ-loaded lysosomes can no longer fuse with autophagosomes, thus blocking autophagy at a late stage $(30,31)$. In this study, CQ not only enhanced erlotinib cytotoxicity, but also induced significant cell death in HCC827, A549, and H1975 cells by single treatment, which was quite different from the effect of the another autophagy inhibitor 3-MA. The distinct mechanisms for blocking autophagy may account for this inconformity. However, studies on cytotoxicity of CQ on cancer cells were not consistent, some claimed that CQ single treatment was able to induce marked cytotoxicity to cancer cells (32-36), but others reported that CQ single treatment showed only modest cytotoxicity $(13,29)$. To explain this contradiction, cell line difference may be an element, while distinct CQ concentration and exposure time may be the main cause.

ER stress, apoptosis and autophagy. ER responds to ER stress through translational attenuation, enhancement of protein folding ability, and degradation of unfolded proteins by a quality-control system. However, when the stress exceeds the ability of ER to process, apoptotic signals are elicited (37-39). CHOP plays an important role in ER stress-induced apoptosis as the fact that $\mathrm{CHOP}^{-/-}$mice exhibit reduced apoptosis in response to ER stress (40). A link between autophagy and ER stress has been substantiated by the finding that the PERKeIF $2 \alpha-C H O P$ pathway is essential for autophagy induction under ER stress, in which CHOP was able to transcriptionally regulate more than a dozen ATG genes $(41,42)$. On the contrary, elicitation of ER stress demonstrated by increased IRE1 activity was observed in ATG-knockout mice, indicating dysregulated autophagy may also trigger ER stress as a feedback mechanism $(43,44)$. In this study, we found that autophagy inhibition by CQ elicited a slight induction of ER stress, demonstrated by increased eIF $2 \alpha$ phosphorylation. Combination of CQ and erlotinib further increased eIF $2 \alpha$ phosphorylation, which induced apoptosis through upregulation of CHOP. This autophagy inhibition-induced ER stress offered a further validation for the ER stress-autophagy feedback paradigm and a new interpretation for the synergistic effect of autophagy inhibition and erlotinib.

In conclusion, the role of autophagy in cancer biology and cancer treatment is complex, and related studies have shown consistent, but also contradictory, conclusions. In this study, we confirmed the ability of erlotinib to induce autophagy in not only EGFR-TKI-sensitive but also EGFR-TKI-resistant cancer cells. Inhibition of autophagy acts synergistically with erlotinib to overcome erlotinib resistance through modulation of ER stress induced apoptosis, in which CHOP acts as a key effector molecule. Together, autophagy is elicited as a cytoprotective process when cancer cells are facing erlotinib exposure, and inhibition of autophagy may represent a promising therapeutic strategy to overcome erlotinib resistance in NSCLC.

\section{Acknowledgements}

This study was supported by grants from the National Natural Science Foundation of China (no. 81302020).

\section{References}

1. Siegel R, Naishadham D and Jemal A: Cancer statistics, 2013. CA Cancer J Clin 63: 11-30, 2013.

2. Sui X, Kong N, Zhu M, Wang X, Lou F, Han W and Pan H: Cotargeting EGFR and autophagy signaling: A novel therapeutic strategy for non-small-cell lung cancer. Mol Clin Oncol 2: 8-12, 2014.

3. Camidge DR, Pao W and Sequist LV: Acquired resistance to TKIs in solid tumours: Learning from lung cancer. Nat Rev Clin Oncol 11: 473-481, 2014.

4. Lee JY, Lim SH, Kim M, Kim S, Jung HA, Chang WJ, Choi MK, Hong JY, Lee SJ, Sun JM, et al: Is there any predictor for clinical outcome in EGFR mutant NSCLC patients treated with EGFR TKIs? Cancer Chemother Pharmacol 73: 1063-1070, 2014.

5. Park S, Langley E, Sun JM, Lockton S, Ahn JS, Jain A, Park K, Singh S, Kim P and Ahn MJ: Low EGFR/MET ratio is associated with resistance to EGFR inhibitors in non-small cell lung cancer. Oncotarget 6: 30929-30938, 2015.

6. Presutti D, Santini S, Cardinali B, Papoff G, Lalli C, Samperna S, Fustaino V, Giannini G and Ruberti G: MET gene amplification and MET receptor activation are not sufficient to predict efficacy of combined MET and EGFR inhibitors in EGFR TKI-resistant NSCLC cells. PLoS One 10: e0143333, 2015.

7. Ricciuti B, Mecca C, Cenci M, Leonardi GC, Perrone L, Mencaroni C, Crinò L, Grignani F, Baglivo S, Chiari R, et al: miRNAs and resistance to EGFR-TKIs in EGFR-mutant non-small cell lung cancer: Beyond 'traditional mechanisms' of resistance. E Cancer Med Sci 9: 569, 2015.

8. Chong CR and Jänne PA: The quest to overcome resistance to EGFR-targeted therapies in cancer. Nat Med 19: 1389-1400, 2013.

9. Amaravadi RK, Lippincott-Schwartz J, Yin XM, Weiss WA, Takebe N, Timmer W, DiPaola RS, Lotze MT and White E: Principles and current strategies for targeting autophagy for cancer treatment. Clin Cancer Res 17: 654-666, 2011.

10. Yang ZJ, Chee CE, Huang S and Sinicrope F: Autophagy modulation for cancer therapy. Cancer Biol Ther 11: 169-176, 2011.

11. Paglin S, Hollister T, Delohery T, Hackett N, McMahill M, Sphicas E, Domingo D and Yahalom J: A novel response of cancer cells to radiation involves autophagy and formation of acidic vesicles. Cancer Res 61: 439-444, 2001.

12. Ertmer A, Huber V, Gilch S, Yoshimori T, Erfle V, Duyster J, Elsässer HP and Schätzl HM: The anticancer drug imatinib induces cellular autophagy. Leukemia 21: 936-942, 2007.

13. Li X and Fan Z: The epidermal growth factor receptor antibody cetuximab induces autophagy in cancer cells by downregulating HIF-1alpha and Bcl-2 and activating the beclin 1/hVps34 complex. Cancer Res 70: 5942-5952, 2010.

14. Han W, Pan H, Chen Y, Sun J, Wang Y, Li J, Ge W, Feng L, Lin $\mathrm{X}$, Wang $\mathrm{X}$, et al: EGFR tyrosine kinase inhibitors activate autophagy as a cytoprotective response in human lung cancer cells. PLoS One 6: e18691, 2011.

15. Suh DH, Kim MK, Kim HS, Chung HH and Song YS: Unfolded protein response to autophagy as a promising druggable target for anticancer therapy. Ann NY Acad Sci 1271: 20-32, 2012.

16. Ron D and Walter P: Signal integration in the endoplasmic reticulum unfolded protein response. Nat Rev Mol Cell Biol 8: 519-529, 2007.

17. Harding HP, Zhang Y, Bertolotti A, Zeng H and Ron D: Perk is essential for translational regulation and cell survival during the unfolded protein response. Mol Cell 5: 897-904, 2000.

18. Zou Y, Ling YH, Sironi J, Schwartz EL, Perez-Soler R and Piperdi B: The autophagy inhibitor chloroquine overcomes the innate resistance of wild-type EGFR non-small-cell lung cancer cells to erlotinib. J Thorac Oncol 8: 693-702, 2013. 
19. $\mathrm{Hu}$ YL, Jahangiri A, Delay M and Aghi MK: Tumor cell autophagy as an adaptive response mediating resistance to treatments such as antiangiogenic therapy. Cancer Res 72: 4294-4299, 2012.

20. Jo GH, Bogler O, Chwae YJ, Yoo H, Lee SH, Park JB, Kim YJ, Kim JH and Gwak HS: Radiation-induced autophagy contributes to cell death and induces apoptosis partly in malignant glioma cells. Cancer Res Treat 47: 221-241, 2015.

21. Sui X, Chen R, Wang Z, Huang Z, Kong N, Zhang M, Han W, Lou F, Yang J, Zhang Q, et al: Autophagy and chemotherapy resistance: A promising therapeutic target for cancer treatment. Cell Death Dis 4: e838, 2013.

22. Sugita S, Ito K, Yamashiro Y, Moriya S, Che XF, Yokoyama T, Hiramoto $M$ and Miyazawa K: EGFR-independent autophagy induction with gefitinib and enhancement of its cytotoxic effect by targeting autophagy with clarithromycin in non-small cell lung cancer cells. Biochem Biophys Res Commun 461: 28-34, 2015

23. Li YY, Lam SK, Mak JC, Zheng CY and Ho JC: Erlotinibinduced autophagy in epidermal growth factor receptor mutated non-small cell lung cancer. Lung Cancer 81: 354-361, 2013.

24. Gupta A, Roy S, Lazar AJ, Wang WL, McAuliffe JC, Reynoso D, McMahon J, Taguchi T, Floris G, Debiec-Rychter M, et al: Autophagy inhibition and antimalarials promote cell death in gastrointestinal stromal tumor (GIST). Proc Natl Acad Sci USA 107: 14333-14338, 2010.

25. Milano V,Piao Y,LaFortune T and de Groot J: Dasatinib-induced autophagy is enhanced in combination with temozolomide in glioma. Mol Cancer Ther 8: 394-406, 2009.

26. Martin AP, Park MA, Mitchell C, Walker T, Rahmani M, Thorburn A, Häussinger D, Reinehr R, Grant S and Dent P: BCL-2 family inhibitors enhance histone deacetylase inhibitor and sorafenib lethality via autophagy and overcome blockade of the extrinsic pathway to facilitate killing. Mol Pharmacol 76: 327-341, 2009

27. Guo GF, Jiang WQ, Zhang B, Cai YC, Xu RH, Chen XX, Wang F and Xia LP: Autophagy-related proteins Beclin-1 and LC3 predict cetuximab efficacy in advanced colorectal cancer. World J Gastroenterol 17: 4779-4786, 2011.

28. Moreira-Leite FF, Harrison LR, Mironov A, Roberts RA and Dive C: Inducible EGFR T790M-mediated gefitinib resistance in non-small cell lung cancer cells does not modulate sensitivity to PI103 provoked autophagy. J Thorac Oncol 5: 765-777, 2010.

29. Tang MC, Wu MY, Hwang MH, Chang YT, Huang HJ, Lin AM and Yang JC: Chloroquine enhances gefitinib cytotoxicity in gefitinib-resistant nonsmall cell lung cancer cells. PLoS One 10: e0119135, 2015.

30. Klionsky DJ, Abeliovich H, Agostinis P, Agrawal DK, Aliev G, Askew DS, Baba M, Baehrecke EH, Bahr BA, Ballabio A, et al: Guidelines for the use and interpretation of assays for monitoring autophagy in higher eukaryotes. Autophagy 4: 151-175, 2008.
31. Morgan MJ, Gamez G, Menke C, Hernandez A, Thorburn J, Gidan F, Staskiewicz L, Morgan S, Cummings C, Maycotte P, et al: Regulation of autophagy and chloroquine sensitivity by oncogenic RAS in vitro is context-dependent. Autophagy 10: 1814-1826, 2014

32. Kimura T, Takabatake Y, Takahashi A and Isaka Y: Chloroquine in cancer therapy: A double-edged sword of autophagy. Cancer Res 73: 3-7, 2013.

33. Hu T, Li P, Luo Z, Chen X, Zhang J, Wang C, Chen P and Dong Z: Chloroquine inhibits hepatocellular carcinoma cell growth in vitro and in vivo. Oncol Rep 35: 43-49, 2016.

34. Fan C, Wang W, Zhao B, Zhang S and Miao J: Chloroquine inhibits cell growth and induces cell death in A549 lung cancer cells. Bioorg Med Chem 14: 3218-3222, 2006.

35. Zheng Y, Zhao YL, Deng X, Yang S, Mao Y, Li Z, Jiang P, Zhao X and Wei Y: Chloroquine inhibits colon cancer cell growth in vitro and tumor growth in vivo via induction of apoptosis. Cancer Invest 27: 286-292, 2009.

36. Yang A, Rajeshkumar NV, Wang X, Yabuuchi S, Alexander BM, Chu GC, Von Hoff DD, Maitra A and Kimmelman AC: Autophagy is critical for pancreatic tumor growth and progression in tumors with p53 alterations. Cancer Discov 4: 905-913, 2014.

37. Oyadomari S and Mori M: Roles of CHOP/GADD153 in endoplasmic reticulum stress. Cell Death Differ 11: 381-389, 2004.

38. Wolff S, Weissman JS and Dillin A: Differential scales of protein quality control. Cell 157: 52-64, 2014.

39. Buchberger A, Bukau B and Sommer T: Protein quality control in the cytosol and the endoplasmic reticulum: Brothers in arms. Mol Cell 40: 238-252, 2010.

40. Oyadomari S, Koizumi A, Takeda K, Gotoh T, Akira S, Araki E and Mori M: Targeted disruption of the Chop gene delays endoplasmic reticulum stress-mediated diabetes. J Clin Invest 109: 525-532, 2002

41. B'chir W, Maurin AC, Carraro V, Averous J, Jousse C, Muranishi Y, Parry L, Stepien G, Fafournoux P and Bruhat A: The eIF2 $\alpha /$ ATF4 pathway is essential for stress-induced autophagy gene expression. Nucleic Acids Res 41: 7683-7699, 2013.

42. Senft D and Ronai ZA: UPR, autophagy, and mitochondria crosstalk underlies the ER stress response. Trends Biochem Sci 40: 141-148, 2015.

43. Deegan S, Saveljeva S, Gorman AM and Samali A: Stressinduced self-cannibalism: On the regulation of autophagy by endoplasmic reticulum stress. Cell Mol Life Sci 70: 2425-2441, 2013.

44. Adolph TE, Tomczak MF, Niederreiter L, Ko HJ, Böck J, Martinez-Naves E, Glickman JN, Tschurtschenthaler M, Hartwig J, Hosomi S, et al: Paneth cells as a site of origin for intestinal inflammation. Nature 503: 272-276, 2013. 